\title{
Spectral and Time-Domain Analyses of Heart-Rate Variability in Children with Severe Upper Airway Obstruction
}

\author{
Berna Şaylan¹, Ayhan Çevik², Vedide Tavlı${ }^{3}$, Ayça Vitrinel ${ }^{4}$ \\ 'Department of Pediatric Cardiology, Dışkapı Children's Hospital, Ankara, Turkey \\ 2Department of Pediatric Cardiology, Faculty of Medicine, Gazi University, Ankara, Turkey \\ ${ }^{3}$ Department of Pediatric Cardiology, Faculty of Medicine, Yeditepe University, İstanbul, Turkey \\ ${ }^{4}$ Department of Pediatrics, Faculty of Medicine, Yeditepe University, İstanbul, Turkey
}

\begin{abstract}
Objective: Heart rate variability (HRV) is a noninvasive index of neural activity of the heart. This study assessed the heart-rate variability response in children with severe upper airway obstruction.

Material and Methods: A prospective trial was carried out in 15 children with severe adenoid and/or tonsil hypertrophy, compared to 15 age matched healthy children in order to attempt to relate such changes. Frequency domain measurements of the high and low frequency bands and the ratio low frequency/high frequency were derived from Holter electrocardiography recordings and computed by Fast Fourier analysis for five minute intervals. Time domain measurements were derived from 24 hour Holter recordings.

Results: All spectral analysis of heart rate variability was altered in both preoperative and postoperative (three months after the operation) recordings compared to the control group. In both groups, time domain indices were significantly lower compared to the control group. Mean R-R values were significantly reduced in pre and postoperative groups compared with control group, with the night time mean $R-R$ values being significantly lower ( $p<0.05)$.
\end{abstract} These results indicate the increased frequency domain parameters in two groups.

Conclusion: In this study, patients demonstrated altered volume loads and autonomic response. Further studies are needed to assess when such cardiac findings normalize upon relief of the upper airway obstruction.

Key Words: Heart-rate variability, children, airway

Received: 10.09.2009

Accepted: 11.12 .2009

\section{Introduction}

In children the most common cause of severe upper airway obstruction and sleep apnea is hypertrophy of the tonsils and adenoids (1). Cardiovascular and pulmonary complications such as right heart failure, pulmonary hypertension and dysrhythmias commonly occur in these children (2). Severe adenoid and tonsil hypertrophy are associated with morbidity and mortality caused by various degrees of obstructive sleep apnea. Even if there is not an apnea syndrome, the patients may have serious side effects. Little is known about heart rate variability in patients with severe upper airway obstruction. It is known that cardiovascular and pulmonary changes can resolve after surgery (3). The cardiac autonomic system controls heart rate by means of parasympathetic and sympathetic activity, and the rate reflects the balance or imbalance of these two influences. Heart rate variability is a noninvasive index of neural activity of the heart. Reduced heart rate variabilty is an increased risk of death in various diseases, with reduced fetal heart rate variability indicating that clinical hypoxia may be developing. The changes of the autonomic nervous system or the cathecolamine secretion is thought to be responsible for the arrhythmogenic effect (4). Impaired cardiac reflexes have been demonstrated in sleep apnea patients by means of spectral heart rate variability analysis. The analysis of timedependent heart rate variability measurements has also been considered useful in the screening of sleep apnea patients. However, the relationship between heart rate variability and the cardiac autonomic dysfunction of obstructive sleep apnea syndrome (OSAS) and hypertrophic tonsils and adenoids is not completely understood. In this study, we aimed to assess the modulation of sympathetic and parasymphatheic effect on the heart in chronic upper airway obstruction and the outcome in the early period following surgery.

\section{Materials and Methods}

We analysed the 24-hour ambulatory electrocardiogram through a prospective follow-up in 15 children $(9$ male, 6 female; with a mean age of $6.3 \pm 1.5$ years) having severe adenoid/tonsilar hypertrophy before and three months following surgical intervention and in 15 healthy age matched children serving as control (6.2 \pm 2.2 years; $p>0.05)$. None of 
the patients had a past clinical history of any other illness (diabetes mellitus, neuropathy, arterial hypertension, pulmonary disease, i.e. conditions that could affect the autonomic nervous syste), and medications that may affect the cardiovascular system. Patients with electrolyte imbalance were excluded from the study. The following were performed for all patients and controls: history and physical examination (auscultation, electrocardiogram, chest X-ray, echocardiography, and Holter ambulatory monitoring).

\section{Heart-Rate Variability Measurement}

A standard ambulatory Holter recording system with a three-channel recorder (Biomedical SystemCentury 2000/3000 Holter monitoring system, version 1.32, (St. Lous, MO) was used for analysis after adjustment of RR intervals manually. Intervals between ectopic beats, between normal and ectopic beats, and intervals inaccurately measured because of the artifacts were excluded. Heart rate variability was assessed in two ways: (1) time-domain analysis and (2) frequency-domain analysis. The mean heart rate, standard deviation of all RR intervals (SDNN), mean standard deviation of NN intervals in 5 -min recordings (SDNNI), the standard deviation of mean NN intervals in 5-min recordings (SDANN), and the root mean square of successive differences (RMSSD) were measured in the time-domain analysis of heart rate variability. Spectral measures were obtained by the fast Fourier transform method. The power in the heart-rate spectrum between 0.003 and $0.40 \mathrm{~Hz}$ was defined as total energy (ms2). This power was divided into two components: low frequency (LF, 0.04-0.15 Hz) and high frequency (HF, 0.16-0.4 Hz), It was believed that high frequency is a marker of solely parasympathetic activity and that low frequency gives mainly a measure of sympathetic activity with some influence from the parasympathetic nervous system (5). In order to differentiate between the influence of the parasympathetic activity on the low frequency spectral power, the ratio of low frequency/high frequency (which is an indicator of sympathovagal balance) was calculated. High values of this ratio indicated dominant sympathetic activity. As in previous reports, many time and frequency-domain variables were strongly correlated with each other $(6,7)$. The spectral and time-domain indexes of heart rate variability were compared between patients with adenoid-tonsilar hypertrophy in the preoperative and postoperative periods and control subjects. The time-domain heart rate variability indexes were compared for an interval of 24 hour.

\section{Statistical Analysis}

Statistical analysis was performed using students't-test utilizing software (SPSS; version 11.0). The association between categorized variables was studied by ki-square and student $t$ test. A p value $<0.05$ was considered statistically significant.

\section{Results}

Fifteen patients (9 male, 6 female; with a mean age of $6.3 \pm 1.5$ years) with severe upper airway obstruction patients due to hypertrophied adenoid and/or tonsils have features as shown in Table 1. Four patients manifested with obstructive sleep apnea (26.7\%). Reduced heart rate variability time parameters, "SDNN", "SDNNi", "SDANN" and "RMSSD" were observed in both preoperative and postoperative recordings compared to the control group $(p<0.05)$ (Table 2, 3). Conversely, patients exhibited an increase in high frequency power, therefore a reduced low frequency / high frequency power ratio favored protective parasympathetic tonus.

\section{Discussion}

Heart rate variation depends on the influence of sympathetic and parasympathetic activity on the sinus node. Age has been shown to be an important determinant of heart rate variability in normal children (6). There were no statistically significant difference between the ages of the patients and the control group in this study. A strong correlation was found between mean R-R interval over the duration of analysis and over five hours of quiet sleep by Massin et al, concluding that correlation between mean $\mathrm{R}-\mathrm{R}$ interval and indices of heart rate variability during sleep did not add important imformation to the correlation of same parameters over 24 hours (7). In this study, 24 hour values of heart rate variability indices were compared to the controls. We found significantly reduced heart rate variability time domain indices ("SDNN", "SDNNi", "SDANN" and "RMSSD") in both the pre and early postoperative recordings compared to the control group. However, the difference between preoperative and early postoperative recordings was not statistically significant. Sleep apnea, as assessed by pulse oxymetry and apnea monitoring, was present in four of the patients, which resolved early after operation. Hypoxia, associated with severe upper airway obstruction may be responsible for the autonomic imbalance and circadian variation.

Sympathovagal balance is best reflected in the ratio of low-to-high frequency components $(8,9)$. The respiratory rate is mildly higher in the first group, however no difference at all exists in the second group suggesting the influence of the sympathetic system rather than respiration. Although indifference exists with low frequency/high frequency values, (being scattered with a high Standard deviation value) parameters of time domain analysis which express long-term heart rate variability, indicate an overall imbalance in the cholinergic and adrenergic activities.

There were some previous studies in which investigators attempted to find a relationship between "OSAS" severity

Table 1. Demographic data of Group 1-2

\begin{tabular}{|lcccc|}
\hline & Preop & Postop & Control & p \\
\hline Age (years) & $6.3 \pm 1.5$ & $6.5 \pm 1.5$ & $6.2 \pm 2.2$ & $\mathrm{NS}$ \\
Weight $(\mathrm{kg})$ & $24 \pm 6$ & $26 \pm 6.4$ & $28.4 \pm 3.6$ & 0.001 \\
Height $(\mathrm{cm})$ & $119 \pm 8.3$ & $122 \pm 8.1$ & $120 \pm 10.9$ & 0.001 \\
Respir. rate $(/ \mathrm{min})$ & 32 & 28 & 28 & $\mathrm{NS}$ \\
$\mathrm{TA}(\mathrm{mmHg})$ & $90 / 50$ & $85 / 50$ & $90 / 40$ & $\mathrm{NS}$ \\
$\mathrm{SaO}_{2} \max (\%)$ & $95.6 \pm 14$ & $98.6 \pm 1.8$ & 99 & 0.02 \\
$\mathrm{SaO}_{2} \min (\%)$ & $91.33 \pm 3.5$ & $91.2 \pm 4.2$ & 95 & $\mathrm{NS}$ \\
\hline
\end{tabular}

NS: non-significant, Preop: preoperative, Postop: postoperative 
Table 2. Mean \pm 1 SD values of HRV time and frequency domain analysis

\begin{tabular}{|lccccc|}
\hline & Preop & Postop & Control & P (pre-control) & P (post-control) \\
\hline SDNN (m/sec) & $106.33 \pm 42.44$ & $101.13 \pm 31.81$ & $161.73 \pm 42$ & 0.003 & 0.001 \\
SDNNi $(\mathrm{m} / \mathrm{sec}$ ) & $59.8 \pm 28.8$ & $58.06 \pm 21.13$ & $88.93 \pm 25.63$ & 0.008 & 0.03 \\
SDANN (m/sec) & $84.26 \pm 33.29$ & $86.46 \pm 25.26$ & $134.53 \pm 40.15$ & 0.001 & 0.001 \\
RMSSD & $50.06 \pm 32.68$ & $50.73 \pm 28.24$ & $89.20 \pm 40.00$ & 0.007 & 0.009 \\
MeanRR & $605.06 \pm 80.9$ & $592.73 \pm 66.9$ & $705.66 \pm 65.61$ & 0.002 & 0.001 \\
DaytimeRR & $554.84 \pm 32$ & $545.32 \pm 23$ & $699.27 \pm 30$ & $<0.05$ & $<0.05$ \\
NighttimeRR & $696.4 \pm 24$ & $653.8 \pm 12$ & $582 \pm 45$ & $<0.05$ & $<0.05$ \\
LF/HF & $2.57 \pm 2.03$ & $2.25 \pm 1.06$ & $2.33 \pm 0.80$ & NS & NS \\
Day time LF/HF & $2.67 \pm 1.4$ & $2.36 \pm 0.7$ & $2.39 \pm 0.8$ & NS & NS \\
Nighttime LF/HF & $2.33 \pm 1.7$ & $2.10 \pm 1.3$ & $2.18 \pm 0.9$ & NS & NS \\
\hline LF: low frequency, HF: high frequency & & & &
\end{tabular}

Table 3. HRV parameters of four patients manifesting sleep apnea

\begin{tabular}{|c|c|c|c|c|c|c|}
\hline & Preop & Postop & Control & $\underset{(1-2)}{p}$ & $\underset{(1-3)}{p}$ & $\underset{(2-3)}{p}$ \\
\hline $\mathrm{LF} / \mathrm{HF}$ & $2.19 \pm 0.58$ & $1.86 \pm 0.28$ & $2.33 \pm 0.80$ & NS & NS & NS \\
\hline SDNN & $117 \pm 42.6$ & $108.5 \pm 31.0$ & $161.73 \pm 42$ & NS & NS & NS \\
\hline
\end{tabular}

and heart rate variability. Aydin and colleagues reported that total power, low frequency, and low frequency/high frequency ratio were higher in patients than those in controls, and that low frequency and low frequency/high frequency ratio were increased in the severe "OSAS" group compared with the mild "OSAS" group (10). In this study, there were no statistically significant difference between pre and postoperative groups and compared to the control group. Reduced heart rate variability parameters observed both preoperatively and early after operation may be considered as an indication for intervention. Yilmaz et al. studied HRV on children having adenotonsillar hypertrophy, and reported that the prevalence of arrhythmias, HRV values were not changed significantly in the post-operative period ( $p>0.05$ ) (11).

However as can be seen clearly from the parameters, there is a complex relationship between the heart rate, heart rate variability and autonomic nervous system in children with severe upper airway obstruction. Lower heart rate variability has been well established in children with apparently life threatening events with obstructive breathing episodes.

In conclusion, significant chronic upper airway obstruction secondary to adenoids and tonsils is accompanied with increase sympathetic tonus (including both daily and nighttime) which does not resolve significantly in the early period after the surgery.

\section{Limitations of this Study}

This study is a single centre, observational and comparative study and the number of cases included in the study is relatively low and the authors suggest confirmation of the findings with a larger number of cases. Polysomnography (PSG) is recognised as the most useful laboratory test to assess the presence and severity of obstructive sleep apnea. Because of lack of a formal sleep laboratory, PSG studies were not performed for any of the patients before or after the operation.

\section{Conflict of Interest}

No conflict of interest was declared by the authors.

\section{References}

1. Lim J, McKean MC. Adenotonsillectomy for obstructive sleep apnoea in children. Cochrane Database Syst Rev 2009;2:CD003136.

2. Duman D, Naiboglu B, Esen HS, Toros SZ, Demirtunc R. Impaired right ventricular function in adenotonsillar hypertrophy. Int J Cardiovasc Imaging 2008;24:261-7.

3. Naiboglu B, Deveci S, Duman D, Kaya KS, Toros S, Kinis V, et al. Effect of upper airway obstruction on pulmonary arterial pressure in children. Int J Pediatr Otorhinolaryngol 2008;72:1425-9.

4. Parati G, Di Rienzo M, Bonsignore MR, Insalaco G, Marrone O, Castiglioni $\mathrm{P}$, et al. Autonomic cardiac regulation in obstructive sleep apnea syndrome: evidence from spontaneous baroreflex analysis during sleep. J Hypertension 1997;15:1621-6.

5. Lucet V, Toumieux MC, Pajot N, Monod N. Paroxysmal vagal hypertonia in infants. Apropos of 14 cases. Arch Fr Pediatr 1984;41:527-31.

6. Longin E, Dimitriadis C, Shazi S, Gerstner T, Lenz T, König S. Autonomic Nervous System Function in Infants and Adolescents: Impact of Autonomic Tests on Heart Rate Variability. Pediatr Cardiol 2009;30:311-24.

7. Massin M, Von Bernuth G. Normal ranges of heart rate variability during infancy and childhood. Pediatr Cardiol 1997;18:297-302.

8. Pagani M, Lombardi F, Guzzetti S, Rimoldi O, Furlan R, Pizzinelli $P$, et al. Power spectral analysis of heart rate and arterial pressure variability as a marker of sympato-vagal interaction in man and conscious dog. Circ Res 1986;59:178-93.

9. Gordon D, Herrera VL, McAlpine L, Cohen RJ, Akselrod R, Lang $P$, et al. Heart rate spectral analysis: a noninvasive probe of cardiovascular regulation in critically ill children with heart disease. Pediatr Cardiol 1988;9:69-77.

10. Aydin M, Altin R, Ozeren A, Kart L, Bilge M, Unalacak M. Cardiac autonomic activity in obstructive sleep apnea: time-dependent and spectral analysis of heart rate variability using 24-hour Holter electrocardiograms. Tex Heart Inst J 2004;31:132-6.

11. Yilmaz F, Gunduz H, Karaaslan K, Arinc H, Cosgun M, Sessiz N, et al. Holter analyses in children with adenotonsillar hypertrophy. International Journal of Pediatric Otorhinolaryngology 2006;8:1443-7. 